\title{
Hepatic Inflammatory Pseudotumor Protruding from the Liver Surface and Directly Penetrating the Colon
}

\author{
Naoki Imazu, Michihiko Shibata, Yudai Koya, Kahori Morino, Yuichi Honma, Michio Senju, \\ Tatsuyuki Watanabe and Masaru Harada
}

\begin{abstract}
:
A 69-year-old man was diagnosed with a liver abscess and received antibiotics at a local hospital. He was referred to our hospital due to a persistent fever. He had hepatic masses protruding from the liver surface toward the transverse colon. We reached a diagnosis of inflammatory pseudotumor (IPT) by a percutaneous liver biopsy. Colonoscopy showed direct invasion of IPT to the colon. His condition improved by the intravenous administration of antibiotics. Hepatic IPT is often misdiagnosed as a malignant tumor. We should consider IPT when we encounter hepatic tumors, and a percutaneous liver biopsy is useful for avoiding unnecessary excessive treatments.
\end{abstract}

Key words: inflammatory pseudotumor, hepatic tumor, liver abscess, liver biopsy

(Intern Med 59: 527-532, 2020)

(DOI: 10.2169/internalmedicine.3599-19)

\section{Introduction}

Inflammatory pseudotumor (IPT), which is also called inflammatory myofibroblastic tumor, is a rare benign disease. Its common organ is the lung, but it has also been observed in the central nervous system, salivary glands, larynx, breast, pancreas, spleen, lymph nodes, skin and liver (1). Hepatic IPT accounts for about $8 \%$ of IPTs among extrapulmonary IPT (2-4), and the incidence was reported to be around $0.7 \%$ among 405 resected liver tumors (5). It is very difficult to make an accurate preoperative diagnosis of hepatic IPT, as it shows nonspecific symptoms and various image findings. We herein report a patient with two hepatic IPTs that protruded from the liver surface and directly penetrated the hepatic flexure of the colon.

\section{Case Report}

A 69-year-old man visited a local hospital after suffering from a fever for two weeks. He had consumed about $100 \mathrm{~g} /$ day of alcohol for 49 years. He had been taking amlodipine besilate since 45 years of age and theophylline and pranlukast hydrate since 60 years of age because of hypertension and chronic obstructive pulmonary disease, respectively. He had no history of diabetes mellitus, a disease that induces ischemia of the liver, cholestatic liver disease, autoimmune diseases (including IgG4-related disease or inflammatory bowel disease), or abdominal surgery. He had been hospitalized three months earlier due to a liver abscess and had undergone percutaneous transhepatic drainage at a local hospital. The drainage had been purulent-like, and Fusobacterium spp. had been detected in bacterial culture, although blood cultures were negative. An examination of the blood showed a white blood cell (WBC) count of $14,900 / \mu \mathrm{L}$ and Creactive protein (CRP) of $16.52 \mathrm{mg} / \mathrm{dL}$. Plain computed tomography (CT) showed 2 consecutive masses with a central low-attenuation area and a maximum size of $6 \mathrm{~cm}$ in diameter in liver segments 5 to 6 (Fig. 1A). He was therefore diagnosed with a liver abscess and emergently admitted to another local hospital (day 1).

Despite treatment with intravenous infusion of antibiotics sulbactam/cefoperazone (SBT/CPZ), the fever did not disappear. He was transferred to our hospital on day 7. On admission, a hard and tender mass was palpable $3 \mathrm{~cm}$ below the costal arch in the right anterior axillary line. Laboratory findings showed a WBC count of $12,300 / \mu \mathrm{L}, \mathrm{CRP}$ of 12.59 $\mathrm{mg} / \mathrm{dL}$, mild elevation of liver enzymes and carcinoembry- 
onic antigen (CEA), and negative for anti-amoebic antibody (Table). After reviewing CT findings on day 1, the border
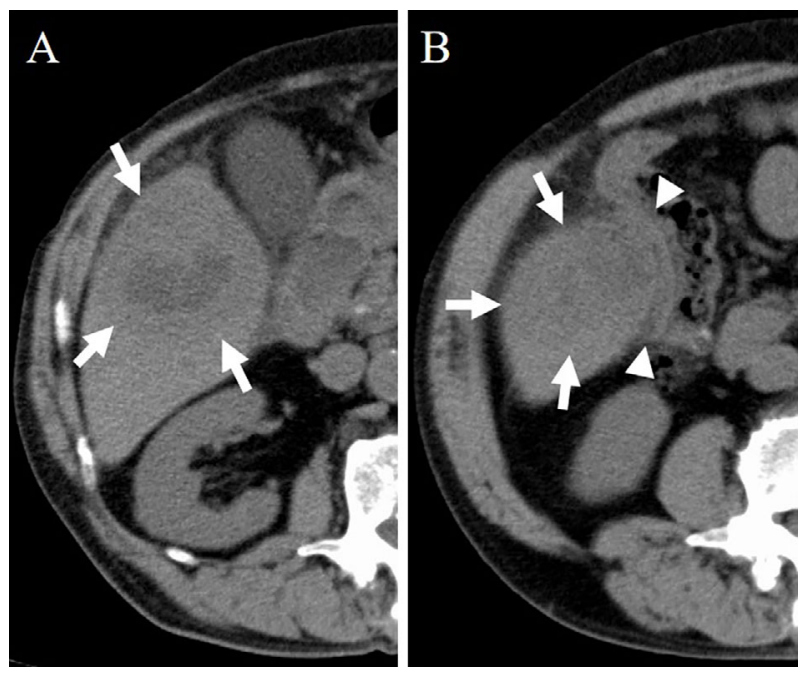

Figure 1. Plain computed tomography (CT) images at a local hospital on day 1 . Two consecutive masses with central low attenuation were observed in liver segments 5 to 6 (arrows). (A) The cranial lesion was $6 \mathrm{~cm}$ in diameter. (B) The border between the caudal mass and hepatic flexure of the colon was illdefined, and the neighboring mesenteric adipose tissue showed stranding (arrowheads). between the caudal mass and hepatic flexure of colon was found to be ill-defined, and the neighboring mesenteric adipose tissue showed stranding (Fig. 1B). On B-mode ultrasonography (US) performed on day 7, the liver lesions were hypoechoic with a central anechoic area (Fig. 2A). On contrast-enhanced US, the lesions were hypervascular at the arterial phase, isovascular at the portal phase, slightly hypovascular at the equilibrium phase (Fig. 2B-D), and hypoechoic at the Kupffer phase. The central areas were avascular at all phases. Contrast-enhanced CT showed that the cranial lesion had increased to $8 \mathrm{~cm}$ in diameter and that both peripheral lesions were slightly enhanced (Fig. 3A). In addition, the caudal lesion protruded from the liver surface, and the border with the colon was indistinct (Fig. 3B).

Although we considered liver abscesses with central necrotic lesion, it was difficult to completely exclude malignant neoplasms, including metastases. Therefore, we performed a US-guided percutaneous aspiration biopsy of the hypoechoic area around the anechoic area of the cranial nodule using a 21-guage needle twice. The section showed infiltration of inflammatory cells, such as lymphoid and plasma cells, and the proliferation of spindle-like fibroblasts that were positive for $\alpha$-smooth muscle actin ( $\alpha$-SMA) according to an immunohistochemical analysis (Fig. 4). We also performed aspiration of the central fluid of the mass and aspirated $50 \mathrm{~mL}$ of purulent-like liquid that was nega-

Table. Laboratory Data on Admission to Our Hospital on Day 7.

\begin{tabular}{lclc}
\hline Hematology & \multicolumn{3}{c}{ Serology } \\
WBC & $12,300 / \mu \mathrm{L}$ & CRP & $12.59 \mathrm{mg} / \mathrm{dL}$ \\
Neutro & $74.8 \%$ & Endotoxin & $<3.0 \mathrm{pg} / \mathrm{mL}$ \\
Lympho & $18.5 \%$ & HBsAg & $(-)$ \\
RBC & $440 \times 10^{4} / \mu \mathrm{L}$ & HCV-Ab & $(-)$ \\
$\mathrm{Hb}$ & $12.1 \mathrm{~g} / \mathrm{dL}$ & Anti-amoebic antibody & $(-)$ \\
Plt & $41.3 \times 10^{4} / \mu \mathrm{L}$ & & \\
& & & \\
Biochemistry & & Coagulation & \\
Alb & $3.2 \mathrm{~g} / \mathrm{dL}$ & PT\% & $82.3 \%$ \\
T-bil & $0.4 \mathrm{mg} / \mathrm{dL}$ & APTT \\
AST & $35 \mathrm{U} / \mathrm{L}$ & Fibrinogen & $39.4 \mathrm{Sec}$ \\
ALT & $37 \mathrm{U} / \mathrm{L}$ & FDP & $851 \mathrm{mg} / \mathrm{dL}$ \\
LDH & $178 \mathrm{U} / \mathrm{L}$ & & $9.6 \mu \mathrm{gg} / \mathrm{mL}$ \\
ALP & $331 \mathrm{U} / \mathrm{L}$ & Tumor maker & \\
$\gamma$ GTP & $174 \mathrm{U} / \mathrm{L}$ & AFP & $2.3 \mathrm{ng} / \mathrm{mL}$ \\
BUN & $10 \mathrm{mg} / \mathrm{dL}$ & CEA & $4.4 \mathrm{ng} / \mathrm{mL}$ \\
Cre & $0.72 \mathrm{mg} / \mathrm{dL}$ & CA19-9 & $6.6 \mathrm{U} / \mathrm{mL}$ \\
FPG & $105 \mathrm{mg} / \mathrm{dL}$ & & \\
HbA1c & $5.8 \%$ & & \\
\hline
\end{tabular}

AFP: alpha-fetoprotein, Alb: albumin, ALP: alkaline phosphatase, ALT: alanine aminotransferase, APTT: activated partial thromboplastin time, AST: aspartate aminotransferase, BUN: blood urea nitrogen, CA19-9 carbohydrate antigen 19-9, CEA: carcinoembryonic antigen, Cre: creatinine, CRP: C-reactive protein, FDP: fibrinogen degradation product, FPG: fast plasma glucose, $\gamma$ GTP: gamma glutamiltranspeptidase, Hb: hemoglobin, HbA1c: hemoglobin A1c, HBsAg: hepatitis B surface antigen, HCV-Ab: hepatitis C virus antibody, LDH: lactate dehydrogenase, Lympho: lymphocyte, Neutro: neutrophils, Plt: platelet, PT: prothrombin time, RBC: red blood cell, T-bil: total bilirubin, WBC: white blood cell 

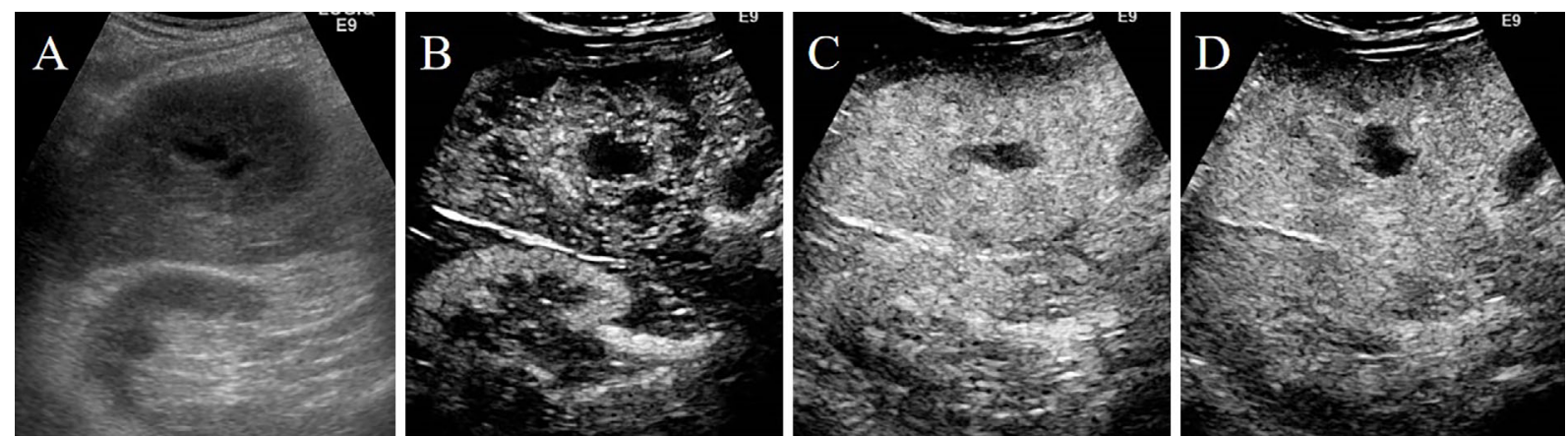

Figure 2. Ultrasonography (US) images on day 7. (A) B-mode US showed the mass as a hypoechoic lesion with a central anechoic area. On contrast-enhanced US, it was hypervascular at the arterial phase $(B)$, isovascular at the portal phase $(C)$ and slightly hypovascular at the equilibrium phase (D). The central areas were avascular at all phases.

tive on bacterial culture and a cytological examination. We therefore made a diagnosis of IPT.

Because the fever and inflammatory reaction persisted, we changed the antibiotics from SBT/CPZ to tazobactam/piperacillin (PIPC/TAZ). The fever and blood inflammation markers immediately improved, and we discontinued PIPC/ TAZ on day 14 (Fig. 5). Total colonoscopy was performed on day 14 because of the CT findings and mild elevation of serum CEA values. It showed a smoothly elevated erythematous lesion at the hepatic flexure (Fig. 6A). A white mass also appeared at the top of the lesion, which was thought to be infiltration from the outside of the colon. We performed 2.8-mm forceps biopsy (Radial $\mathrm{Jaw}^{\mathrm{TM}}$ 4; Boston Scientific, Boston, USA). However, it was difficult to obtain an adequate pathological specimen from the white mass, as it was composed of fibrotic scleroid tissue. The specimen from the erythematous membrane around the white mass showed mild-to-moderate chronic inflammatory changes and epithelial regeneration. Finally, we diagnosed the patient with hepatic IPT with bacterial infection and direct penetration to the hepatic flexure of the colon. CT on day 18 showed that the hepatic IPTs had decreased in size and that the border with the colon had become clear (Fig. 3C, D). He was discharged from our hospital on day 21. The IPTs had almost completely disappeared on CT (Fig. 3E, F), and the colonic lesion was completely healed on colonoscopy two months after the discharge (Fig. 6B).

\section{Discussion}

IPT was first described in the lung in 1939 (6), and hepatic IPT was first reported in 1953 by Pack and Baker (7). The epidemiology of hepatic IPT remains unclear because it is a rare disease. Some previous studies from Asia, Europe and the USA have reported that men accounted for about two-thirds of total hepatic IPT cases $(4,8,9)$. Although the common age described in those studies ranged widely, from the 30 s to 60 s, one study from Korea in 45 cases of histologically proven hepatic IPT reported a median age of 65 years old (9), and our patient was also near this age.
The present patient showed a fever and elevation of serum inflammatory markers and hepatobiliary enzymes. The most common symptoms of hepatic IPT are abdominal pain, a fever, malaise, and weight loss $(4,5,9,10)$. However, about $20 \%$ of the patients are asymptomatic. In addition, the most common abnormal laboratory findings were elevation of serum inflammatory markers, including the erythrocyte sedimentation rate (ESR), CRP level and leukocyte count $(2,9,11)$. Mild elevation of hepatobiliary enzymes is sometimes observed. However, these symptoms and laboratory findings are nonspecific and are also seen in patients with other hepatobiliary infections and malignancies.

The etiology of hepatic IPT remains unknown. However, several factors, such as infection, trauma, biliary obstruction and cholangitis, autoimmune disorders, hepatitis B virus, hepatitis $\mathrm{C}$ virus, IgG4-related cholangitis and primary sclerosing cholangitis, are thought to be closely related to hepatic IPT (12-14). The present case had undergone percutaneous transhepatic liver abscess drainage six months earlier. This traumatic procedure may have been related to his hepatic IPT. In addition, hepatic IPT in the present case may have been caused by liver abscess, as purulent-like liquid was detected in the central lesion, and blood examinations showed marked inflammation. Unfortunately, we did not examine the autoantibody, IgG and IgG4 presence. There were no biliary or pancreatic abnormalities on imaging or physical findings suggesting autoimmune disease. Although he was not an immunocompromised host, we should have considered the possibility of cytomegalovirus (CMV) infectious liver abscess. An instructive report described a case of CMV colitis affecting an immunocompetent patient complicated with liver abscess and subsequent colorectal stenosis requiring surgical resection (15).

It has been reported that most cases with hepatic IPT were diagnosed after surgical resection (14). This is because of the lack of accurate diagnostic imaging methods. The US findings of hepatic IPT show various echogenicity patterns or lesions mimicking an abscess or a hepatocellular carcinoma (16). On contrast-enhanced CT, hepatic IPT often shows late enhancement of the peripheral region (3). How- 

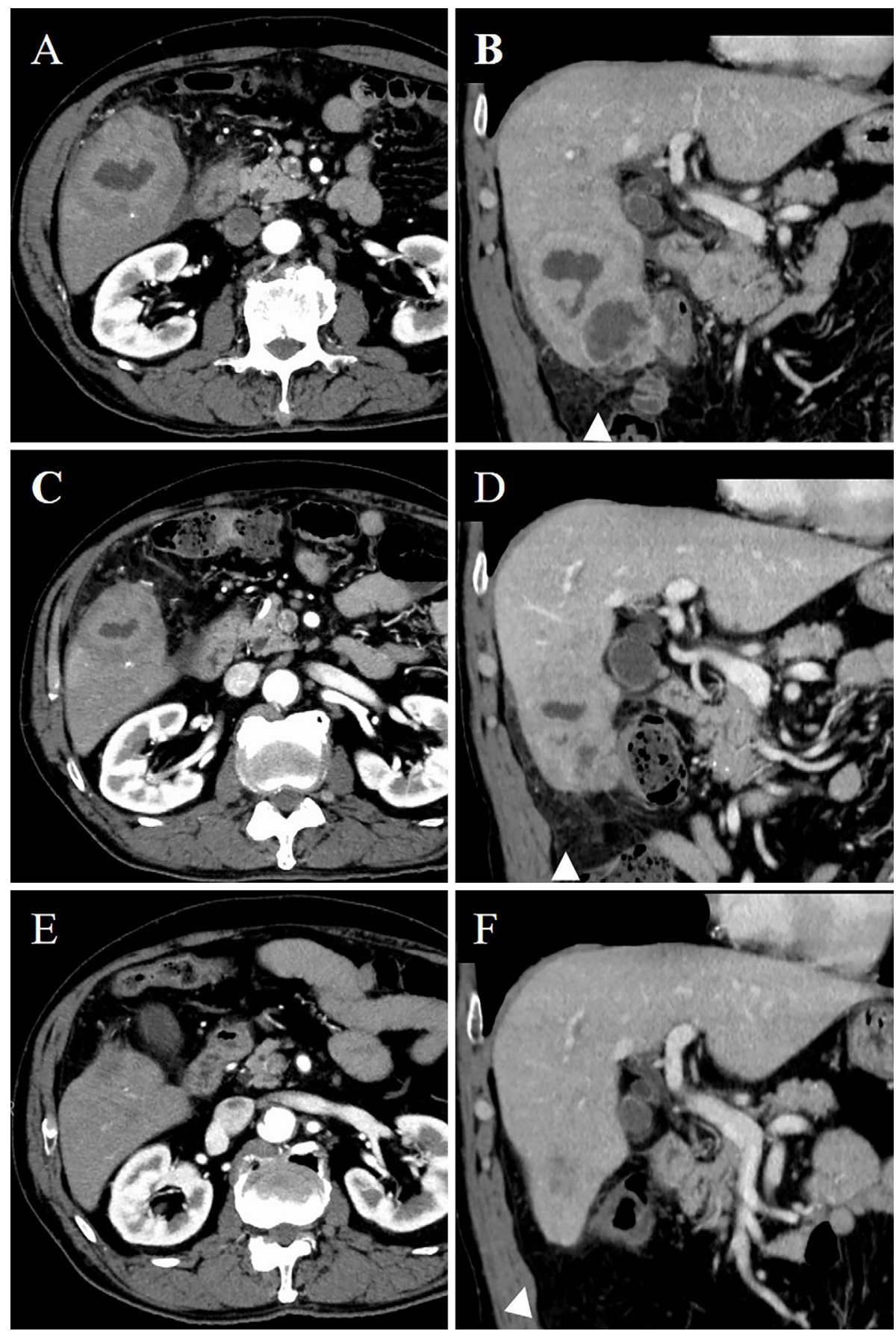

Figure 3. Contrast-enhanced CT images. (A, B) On day 7, the cranial lesion had increased to $8 \mathrm{~cm}$ in diameter and was slightly enhanced with non-enhanced low attenuation foci in the center. The caudal lesion protruded from the liver surface, and the border with the colon was indistinct (arrow). (C, D) On day 18, the two lesions had decreased in size. However, the border with the colon became slightly clearer (arrow). (E, F) Two months after the discharge, the two liver lesions had almost disappeared, and the border with the colon had become clear (arrow).

ever, this enhancement pattern is also seen in adenocarcinoma, such as cholangiocarcinoma or metastatic tumors. In addition, one study found that hepatic IPT sometimes showed early enhancement with washout at the late phase, like hepatocellular carcinoma (17). In the present case, the mass was enhanced from the arterial to equilibrium phase on contrast-enhanced US. The image findings of hepatic IPT are thus variable and nonspecific. This is because the en- hancement patterns created by the contrast medium represent the distribution proportions of inflammatory cells and fibrosis, and this distribution varies depending on the cause and inflammatory phase (18).

Hepatic IPT is usually a single lesion localized to the liver and does not protrude from the liver surface. In rare cases, multiple hepatic IPTs are reported $(4,9)$. In addition, there are some case reports of hepatic IPT extrahepatic 
growth patterns mimicking the present patient (19-21). The present case also had two lesions that directly invaded the colon, mimicking malignant tumors. These findings made it difficult for us to discriminate hepatic IPT from malignant tumors.

The typical pathological findings of IPT are the presence of myofibroblastic spindle cells, plasma cells, macrophages, and lymphocytes without cellular atypia or atypical mitotic

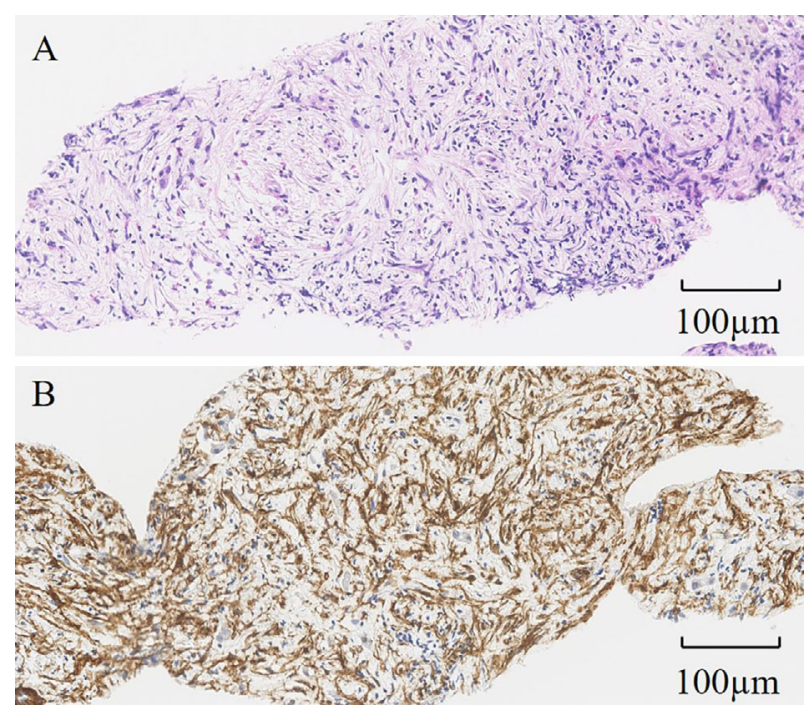

Figure 4. Histopathological findings of the cranial nodule. (A) Hematoxylin and eosin staining showed infiltration of inflammatory cells, such as lymphoid and plasma cells, and proliferation of spindle-like cells. (B) The spindle-like cells were positive on $\alpha$-SMA immunohistochemical staining. figures (22). These findings differ markedly from those of liver abscess and malignant tumors. Therefore, differentiation between hepatic IPT and other liver diseases seems to be easy. However, many cases with hepatic IPT are diagnosed postoperatively rather than preoperatively (14). Although a US-guided percutaneous biopsy plays an important role in making a diagnosis of liver lesions in general, its diagnostic accuracy regarding hepatic IPT is insufficient (23). The major reason for this lack of accuracy is thought to be sampling error. In most cases, hepatic IPT regresses spontaneously or after the administration of antibiotics. We believe that performing a US-guided percutaneous biopsy twice in the hypoechoic area, where viable tumor cells might remain, rather than the anechoic area helped avoid sampling error.

Surgical resection is a treatment option, but unnecessary resection should be avoided (24). A re-biopsy should therefore be considered if the histological findings fail to clearly distinguish among hepatic IPT, cholangiocarcinoma and metastases.

It is difficult to diagnose hepatic IPT because its symptoms are nonspecific and its imaging findings can mimic malignant tumors. In the present case, the spread of inflammation to the hepatic flexure on colonoscopy was noted, and np malignant tissue was detected by a colon biopsy. Colon cancer and liver abscess without liver metastasis have been reported to be simultaneously detected (25). Given the possibility of a discrepancy between the histopathology of the liver and the colon, it is important to perform both a liver and colon biopsy. We reached a diagnosis of hepatic IPT and avoided invasive surgical resection by performing a liver and colon biopsy with a careful immunohistochemical

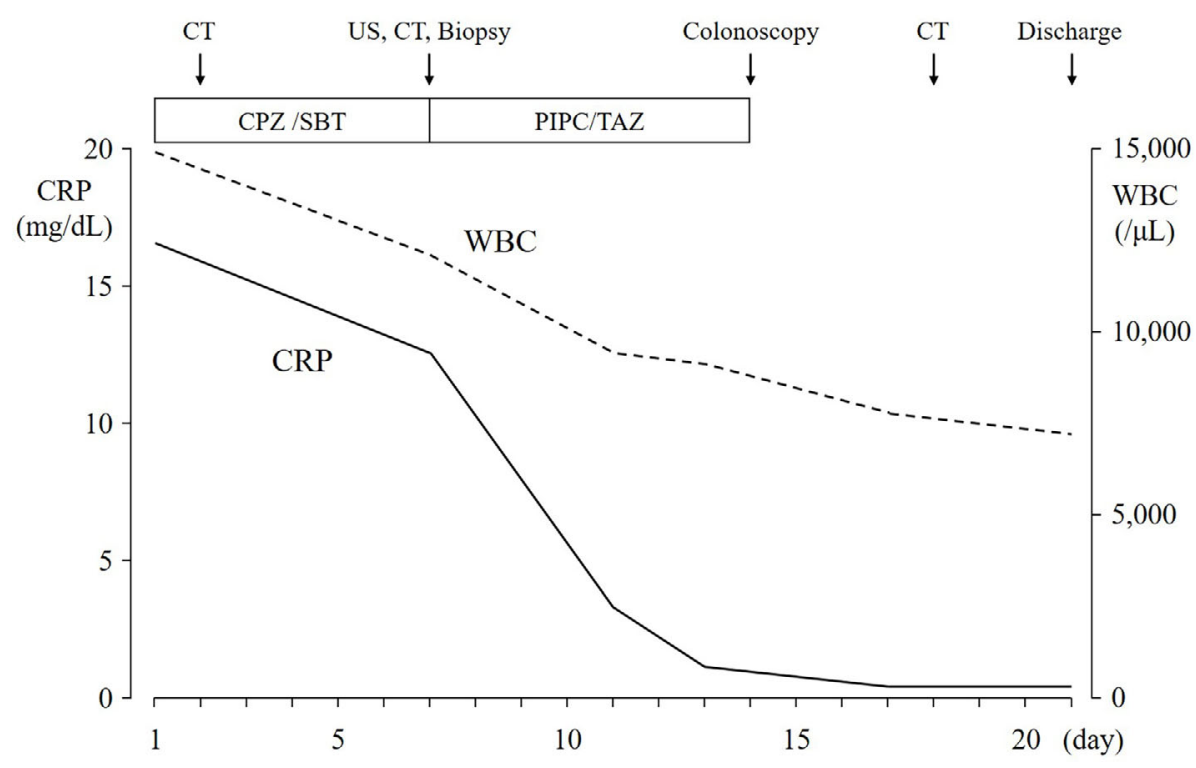

Figure 5. Clinical course. The patient was admitted to a local hospital on day 1 and treated with sulbactam/cefoperazone (SBT/CPZ) from days 1 to 6 . He was transferred to our hospital, and a percutaneous aspiration biopsy was performed on day 7. Tazobactam/piperacillin (PIPC/TAZ) was administered from days 7 to 14. Blood inflammatory markers, such as the white blood cell (WBC) count and C-reactive protein (CRP) level, improved immediately. CT: computed tomography, US: ultrasonography 


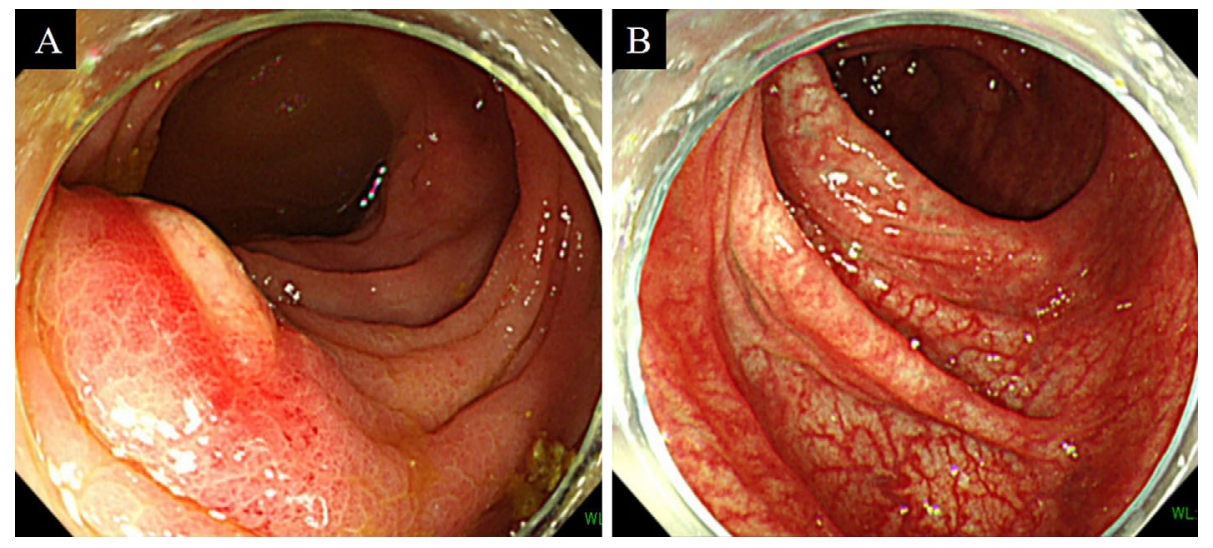

Figure 6. Colonoscopy images at the hepatic flexure of the colon. (A) On day 14, a hard, white mass infiltrated from the outside of the colon. (B) The colonic lesion had completely healed by two months after the discharge.

analysis.

The authors state that they have no Conflict of Interest (COI).

\section{References}

1. Coffin CM, Humphrey PA, Dehner LP. Extrapulmonary inflammatory myofibroblastic tumor: a clinical and pathological survey. Semin Diagn Pathol 15: 85-101, 1998.

2. Horiuchi R, Uchida T, Kojima T, Shikata T. Inflammatory pseudotumor of the liver. Clinicopathologic study and review of the literature. Cancer 65: 1583-1590, 1990.

3. Fukuya $T$, Honda $H$, Matsumata $T$, et al. Diagnosis of inflammatory pseudotumor of the liver: value of CT. AJR Am J Roentgenol 163: 1087-1091, 1994.

4. Tang L, Lai EC, Cong WM, et al. Inflammatory myofibroblastic tumor of the liver: a cohort study. World J Surg 34: 309-313, 2010.

5. Torzilli G, Inoue $\mathrm{K}$, Midorikawa $\mathrm{Y}$, Hui AM, Takayama $\mathrm{T}$, Makuuchi M. Inflammatory pseudotumors of the liver: prevalence and clinical impact in surgical patients. Hepatogastroenterology 48: 1118-1123, 2001

6. Copin MC, Gosselin BH, Ribet ME. Plasma cell granuloma of the lung: difficulties in diagnosis and prognosis. Ann Thorac Surg 61: 1477-1482, 1996.

7. Pack GT, Baker HW. Total right hepatic lobectomy; report of a case. Ann Surg 138: 253-258, 1953.

8. Schmid A, Janig D, Bohuszlavizki A, Henne-Bruns D. Inflammatory pseudotumor of the liver presenting as incidentaloma: report of a case and review of the literature. Hepatogastroenterology 43: 1009-1014, 1996.

9. Park JY, Choi MS, Lim YS, et al. Clinical features, image findings, and prognosis of inflammatory pseudotumor of the liver: a multicenter experience of 45 cases. Gut Liver 8: 58-63, 2014.

10. Goldsmith PJ, Loganathan A, Jacob M, et al. Inflammatory pseudotumours of the liver: a spectrum of presentation and management options. Eur J Surg Oncol 35: 1295-1298, 2009.

11. Koea JB, Broadhurst GW, Rodgers MS, McCall JL. Inflammatory pseudotumor of the liver: demographics, diagnosis, and the case for nonoperative management. J Am Coll Surg 196: 226-235, 2003.

12. Nakanuma Y, Tsuneyama K, Masuda S, Tomioka T. Hepatic inflammatory pseudotumor associated with chronic cholangitis: report of three cases. Hum Pathol 25: 86-91, 1994.

13. Toda K, Yasuda I, Nishigaki Y, et al. Inflammatory pseudotumor of the liver with primary sclerosing cholangitis. J Gastroenterol
35: 304-309, 2000

14. Bae SK, Abiru S, Kamohara Y, et al. Hepatic inflammatory pseudotumor associated with xanthogranulomatous cholangitis mimicking cholangiocarcinoma. Intern Med 54: 771-775, 2015.

15. Soares JB, Ferreira A, Goncalves R, et al. Hepatic abscess and colonic stenosis: two complications of an unlikely cause. Gastroenterology 141: e9-e10, 2011.

16. Walsh SV, Evangelista F, Khettry U. Inflammatory myofibroblastic tumor of the pancreaticobiliary region: morphologic and immunocytochemical study of three cases. Am J Surg Pathol 22: 412-418, 1998.

17. Akatsu T, Wakabayashi G, Tanimoto A, Kameyama K, Kitajima M. Inflammatory pseudotumor of the liver masquerading as hepatocellular carcinoma after a hepatitis B virus infection: report of a case. Surg Today 36: 1028-1031, 2006.

18. Onieva-Gonzalez FG, Galeano-Diaz F, Matito-Diaz MJ, Lopez-Guerra D, Fernandez-Perez J, Blanco-Fernandez G. Inflammatory pseudotumour of the liver. Importance of intra-operative histopathology. Cir Cir 83: 151-155, 2015.

19. Chen CB, Chou CT, Hsueh C, Lee KW, Chen YL. Hepatic inflammatory pseudotumor mimicking hepatocellular carcinoma. J Chin Med Assoc 76: 299-301, 2013.

20. Obana T, Yamasaki S, Nishio K, Kobayashi Y. A case of hepatic inflammatory pseudotumor protruding from the liver surface. Clin J Gastroenterol 8: 340-344, 2015.

21. Honmyo N, Kobayashi T, Tashiro H, et al. Inflammatory pseudotumor of the liver occurring during the course of hepatitis C virusrelated hepatocellular carcinoma treatment: a case report. Int J Surg Case Rep 20: 96-100, 2016.

22. Iguchi $\mathrm{H}$, Yamazaki $\mathrm{H}$, Tsunoda $\mathrm{H}$, Takahashi Y, Yokomori H. A case of inflammatory pseudotumor of the liver mimicking hepatocellular carcinoma on EOB-MRI and PET. Case Rep Med 2013: 594254, 2013.

23. Lupovitch A, Chen R, Mishra S. Inflammatory pseudotumor of the liver. Report of the fine needle aspiration cytologic findings in a case initially misdiagnosed as malignant. Acta Cytol 33: 259-262, 1989.

24. Gluszek S, Kot M, Czerwaty M. Inflammatory pseudotumor of the liver treated surgically. Hepatogastroenterology 46: 2959-2960, 1999.

25. Shigefuku R, Watanabe T, Kanno Y, et al. Fusobacterium nucleatum detected simultaneously in a pyogenic liver abscess and advanced sigmoid colon cancer. Anaerobe 48: 144-146, 2017.

The Internal Medicine is an Open Access journal distributed under the Creative Commons Attribution-NonCommercial-NoDerivatives 4.0 International License. To view the details of this license, please visit (https://creativecommons.org/licenses/ by-nc-nd/4.0/).

(C) 2020 The Japanese Society of Internal Medicine Intern Med 59: 527-532, 2020 